\title{
HLA-matched sibling transplantation with G-CSF mobilized PBSCs and BM decreases GVHD in adult patients with severe aplastic anemia
}

\author{
Sun Zi-Min*, Liu Hui-Lan, Geng Liang-Quan, Wang Xin-Bing, Yao Wen, Liu Xin, Ding Kai-Yang, Han Yong-Sheng, \\ Yang Hui-Zhi, Tang Bo-lin, Tong Juan, Zhu Wei-Bo, Wang Zu-Yi
}

\begin{abstract}
Background: Allogeneic hematopoietic stem cell transplantation (allo-HSCT) is an effective treatment for severe aplastic anemia (SAA). However, graft failure and graft-versus-host disease (GVHD) are major causes of the early morbidity in Allo-HSCT.

Methods: To reduce graft failure and GVHD, we treated fifteen patients with SAA using high- dose of HSCT with both G-CSF mobilized PB and BMSCs from HLA-identical siblings to treat patients with SAA.

Results: All patients had successful bone marrow engraftment. Only one patient had late rejection. Median time to ANC greater than $0.5 \times 10^{9} / \mathrm{L}$ and platelet counts greater than $20 \times 10^{9} / \mathrm{L}$ was 12 and 16.5 days, respectively. No acute GVHD was observed. The incidence of chronic GVHD was 6.67\%. The total three-year probability of diseasefree survival was $79.8 \%$.
\end{abstract}

Conclusion: HSCT with both G-CSF mobilized PB and BMSCs is a promising approach for heavily transfused and/or allo-immunized patients with SAA.

\section{To the Editor}

Allogeneic hematopoietic stem cell transplantation (alloHSCT) is a cure for patients with severe aplastic anemia (SAA). However, complications such as graft failure and graft-versus-host disease (GVHD) have limited the application of allo-HSCT [1,2]. Increasing the number of donor blood stem cells decreases graft failure, however, highdose of blood stem cell transplantation also increases the incidence of GVHD[3]. A combination of un-manipulated marrow and T-cell depleted PBSC as the stem cell source for SAA have shown rapid engraftment without increasing the risk of GVHD [4,5]. Here, we report that transplantation of un-manipulated peripheral blood stem cells (PBSC) combined with bone marrow stem cells (BMSC) in patients with SAA to reduce the incidence of graft failure without negative effects on GVHD.

Fifteen SAA patients, received HLA- 6/6-identical sibling G-CSF-mobilized PB plus BMSC transplantation

\footnotetext{
* Correspondence: zmsun2003@yahoo.com.cn
Department of Hematology, Anhui Medical University Affiliated Anhui

* Correspondence: zmsun2003@yahoo.com.cn
Department of Hematology, Anhui Medical University Affiliated Anhui Provincial Hospital, Hefei, China
}

(Table 1). CY/ALG (12/15 patients) or Flu/CY/ALG (3/15 patients) were used as conditioning regimen for all of them. CsA-MMF regimen was used to prevent aGVHD. Other supportive treatment were also given, such as acyclovir, intravenous rhG-CSF, and intravenous immunoglobulin. The engraftment of transplant cells was determined using the following methods: STR-PCR analysis, Y PCR analysis, and tests for hematopoietic reconstitution and GVHD.

All fifteen patients receiving allo-HSCT had successful bone marrow engraftment except for one of them had a late rejection. The incidence of acute and chronic GVHD was $0 \%$ and $6.67 \%$. The reasons for the decreased incidence may be multifactorial, the use of G-CSF mobilized $\mathrm{PBSC}+\mathrm{BMSC}_{S}$ as the source of grafts, usage of ALG in conditioning regimen and CsA/MMF for the prophylaxis of GVHD. No recipients died from treatment-related complications within the first 100 days after transplantation. There were twelve disease-free survivals. The total three-year probability of disease-free survival was $79.8 \%$ (Figure 1). 
Table 1 Outcome of 15 SAA patients who received the $\mathrm{PB}+\mathrm{BM}$ transplantation

\begin{tabular}{|c|c|c|c|c|c|c|c|c|c|c|c|c|}
\hline \multirow[t]{3}{*}{ No. } & \multirow[t]{3}{*}{ Disease } & \multirow{3}{*}{$\begin{array}{l}\text { Conditioning } \\
\text { Regimen }\end{array}$} & \multirow{3}{*}{$\begin{array}{l}\text { GVHD } \\
\text { Prophylaxis }\end{array}$} & \multicolumn{2}{|c|}{ Cell number } & \multicolumn{3}{|c|}{ Engraftment (days) } & \multirow[b]{3}{*}{$\begin{array}{l}\text { Acute } \\
\text { GVHD }\end{array}$} & \multirow[b]{3}{*}{$\begin{array}{l}\text { chronic } \\
\text { GVHD }\end{array}$} & \multirow[b]{3}{*}{$\begin{array}{l}\text { Survival } \\
\text { (Month) }\end{array}$} & \multirow[b]{3}{*}{$\begin{array}{l}\text { Cause } \\
\text { of } \\
\text { death }\end{array}$} \\
\hline & & & & \multirow{2}{*}{$\begin{array}{l}\mathrm{NC} \times 10^{8} / \mathrm{kg} \\
\mathrm{PB} / \mathrm{BM}\end{array}$} & \multirow{2}{*}{$\begin{array}{l}\mathrm{CD} 34 \times 10^{6} / \mathrm{kg} \\
\mathrm{PB} / \mathrm{BM}\end{array}$} & \multirow{2}{*}{$\begin{array}{l}\text { ANC } \\
>0.5 \\
\times \\
10^{9} / \mathrm{L}\end{array}$} & \multicolumn{2}{|c|}{ PLt } & & & & \\
\hline & & & & & & & $\begin{array}{l}>20 \times \\
10^{9} / L\end{array}$ & $\begin{array}{l}>50 \\
\times \\
10^{9} / \mathrm{L}\end{array}$ & & & & \\
\hline 1 & VSAA-I & CY/ALG & $\mathrm{Cs} A+M M F$ & $5.95 / 3.06$ & $3.07 / 0.89$ & 11 & 15 & 18 & $\mathrm{~N}$ & skin & $80^{+}$ & \\
\hline 2 & VSAA-I & CY/ALG & $\mathrm{CsA}+\mathrm{MMF}$ & $2.47 / 1.9$ & $2.39 / 0.7$ & 11 & 14 & 18 & $\mathrm{~N}$ & $\mathrm{~N}$ & $62^{+}$ & \\
\hline \multirow[t]{2}{*}{3} & SAA-II & CY/ALG & $\mathrm{CsA}+\mathrm{MMF}$ & $2.91 / 2.6$ & $2.33 / 1.48$ & 15 & 47 & 53 & & & 7 & $\begin{array}{l}\text { Late } \\
\text { graft }\end{array}$ \\
\hline & & & & & & & & & $\mathrm{N}$ & $\mathrm{N}$ & & Rejection \\
\hline 4 & VSAA-I & $\mathrm{CY} / \mathrm{ALG}$ & $\mathrm{CsA}+\mathrm{MMF}$ & $2.46 / 2.21$ & $5.66 / 0.95$ & 14 & 22 & 34 & $\mathrm{~N}$ & $\mathrm{~N}$ & $54^{+}$ & \\
\hline 5 & SAA-I & $\mathrm{CY} / \mathrm{ALG}$ & $\mathrm{CsA}+\mathrm{MMF}$ & $6.47 / 1.88$ & $5.3 / 0.47$ & 10 & 20 & 50 & $\mathrm{~N}$ & $\mathrm{~N}$ & 9 & Infection \\
\hline 6 & SAA-I & CY/ALG & $\mathrm{Cs} A+\mathrm{MMF}$ & $4.54 / 3.87$ & $2.81 / 1.1$ & 12 & 20 & 32 & $\mathrm{~N}$ & $\mathrm{~N}$ & $46^{+}$ & \\
\hline 7 & VSAA-I & CY/ALG & $\mathrm{CsA}+\mathrm{MMF}$ & $6.17 / 1.0$ & $1.54 / 0.3$ & 14 & 30 & 35 & $\mathrm{~N}$ & $\mathrm{~N}$ & $30^{+}$ & \\
\hline 8 & SAA-I & CY/ALG & $\mathrm{Cs} A+\mathrm{MMF}$ & $4.64 / 1.86$ & $4.45 / 0.71$ & 11 & 15 & 18 & $\mathrm{~N}$ & $\mathrm{~N}$ & $30^{+}$ & \\
\hline 9 & SAA-II & $\mathrm{Flu} / \mathrm{CY} / \mathrm{ALG}$ & $\mathrm{CsA}+\mathrm{MMF}$ & $5.05 / 1.14$ & $1.36 / 0.33$ & 12 & 17 & 20 & $\mathrm{~N}$ & $\mathrm{~N}$ & $29^{+}$ & \\
\hline 10 & SAA-II & $\mathrm{Flu} / \mathrm{CY} / \mathrm{ALG}$ & $\mathrm{Cs} A+M M F$ & $3.75 / 1.47$ & $4.2 / 0.66$ & 12 & 15 & 16 & $\mathrm{~N}$ & $\mathrm{~N}$ & $28^{+}$ & \\
\hline 11 & SAA-I & CY/ALG & $\mathrm{CsA}+\mathrm{MMF}$ & 2.98/1.77 & $6.62 / 0.9$ & 10 & 15 & 20 & $\mathrm{~N}$ & $\mathrm{~N}$ & $26^{+}$ & \\
\hline 12 & VSAA-I & $\mathrm{CY} / \mathrm{ALG}$ & $\mathrm{CsA}+\mathrm{MMF}$ & $7.80 / 2.6$ & $5.7 / 0.85$ & 12 & 14 & 15 & $\mathrm{~N}$ & N & $26^{+}$ & \\
\hline 13 & SAA-II & $\mathrm{Flu} / \mathrm{CY} / \mathrm{ALG}$ & $\mathrm{Cs} A+\mathrm{MMF}$ & $5.86 / 2.1$ & $5.03 / 0.92$ & 13 & 16 & 16 & $\mathrm{~N}$ & $\mathrm{~N}$ & $20^{+}$ & \\
\hline 14 & VSAA-I & CY/ALG & $\mathrm{Cs} A+\mathrm{MMF}$ & $2.15 / 1.9$ & $0.49 / 1.14$ & 23 & 27 & 35 & $\mathrm{~N}$ & $\mathrm{~N}$ & 5 & Infection \\
\hline 15 & SAA-I & $\mathrm{CY} / \mathrm{ALG}$ & $\mathrm{CsA}+\mathrm{MMF}$ & $8.3 / 0.77$ & $1.66 / 0.17$ & 16 & 29 & 48 & $\mathrm{~N}$ & $\mathrm{~N}$ & $7^{+}$ & \\
\hline \multicolumn{2}{|c|}{$\begin{array}{l}\text { Median } \\
\text { (range) }\end{array}$} & & & $\begin{array}{l}4.64(2.15-8.3) / 1.9 \\
(0.77-3.87) \times \\
10^{8} / \mathrm{kg}\end{array}$ & $\begin{array}{l}3.07(0.49-6.62) / \\
0.85(0.17-1.48) \times \\
106 / \mathrm{kg}\end{array}$ & $\begin{array}{l}\text { Day } \\
12 \\
(10- \\
23)\end{array}$ & $\begin{array}{l}\text { Day } \\
16.5 \\
(14-47)\end{array}$ & $\begin{array}{l}\text { Day } \\
20 \\
(15- \\
53)\end{array}$ & & & $\begin{array}{l}\text { Month } \\
27 \text { (7-80) }\end{array}$ & \\
\hline
\end{tabular}

CY: cyclophosphamide; ALG: antihuman T-lymphocyte globulin; MMF: mycophenolate mofetil; CsA: cyclosporine A; N: without any acute GVHD or chronic GVHD.

Our data indicate that high- dose of HSCT with both G-CSF mobilized PB and BMSCs resulted in a quick engraftment, low graft rejection, a relatively low incidence of acute GVHD, and good DFS, although larger scale, prospective, and randomized studies are required to confirm these benefits.

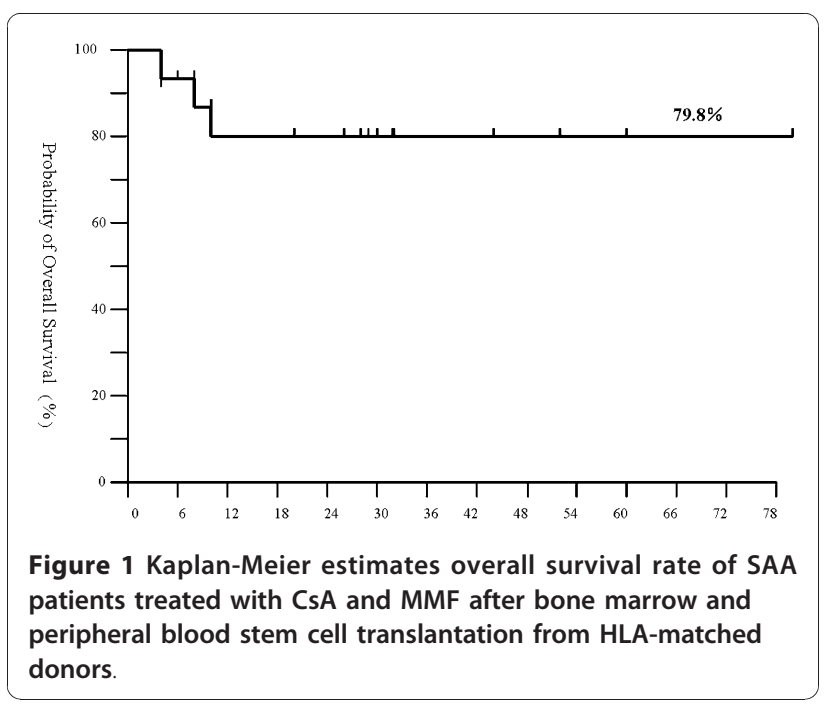

List of abbreviations

allo-HSCT: Allogeneic hematopoietic stem cell transplantation; SAA: severe aplastic anemia; GVHD: graft-versus-host disease; ANC: absolute neutrophil count; MSCs: mesenchymal stem cells; MPCs: mesenchymal (stroma) progenitor cells.

\section{Acknowledgements}

We thank Lijun Xia from Oklahoma Medical Research Foundation for helpful suggestions.

\section{Authors' contributions}

SZM have made substantial contributions to conception and design; LHL, GLQ and WXB participated in the acquisition of data; WZY and ZWB participated in analysis and interpretation of data; YW and TJ drafted the manuscript; HYS, YHZ and LX revising it critically for important intellectual content; DKY and TBL performed the statistical analysis.

All authors have read and approved the final manuscript.

\section{Competing interests}

The authors declare that they have no competing interests.

Received: 19 November 2010 Accepted: 31 December 2010 Published: 31 December 2010

\section{References}

1. Remberger M, Naseh N, Aschan J, et al: G-CSF given after haematopoietic stem cell transplantation using HLA-identical sibling donors is associated to a higher incidence of acute GVHD II-IV. Bone Marrow Transplant 2003, 32(2):217-23.

2. Storey JA, Rebecca F Connor, Zachary T Lewis, David Hurd, Gregory Pomper, Yi K Keung, Manisha Grover, James Lovato, Suzy V Toirti, Frank M Torti, Istvan Molnar: The transplant iron score as a predictor of stem cell transplant survival. Journal of Hematology \& Oncology 2009, 2:44. 
3. Dvorak CC, Gilman AL, Horn B, et al: Primary graft failure after umbilical cord blood transplant rescued by parental haplocompatible stem cell transplantation. J Pediatr Hematol Oncol 2009, 31(4):300-3.

4. Min CK, Kim DW, Lee JW, et al: Supplemental peripheral blood stem cells to decrease marrow rejection in adult patients with severe aplastic anemia. Am J Hematol 2002, 69(1):15-22.

5. Cho BS, Eom KS, Kim YJ, et al: HLA-matched sibling transplantation with $\mathrm{BM}$ and CD34(+)-purified PBSCs in adult patients with high-risk severe aplastic anemia to overcome graft rejection without an increase in GVHD. Bone Marrow Transplant 2010, 11:1-5.

doi:10.1186/1756-8722-3-51

Cite this article as: Zi-Min et al:: HLA-matched sibling transplantation with G-CSF mobilized PBSCs and BM decreases GVHD in adult patients with severe aplastic anemia. Journal of Hematology \& Oncology 2010 3:51.

\section{Submit your next manuscript to BioMed Central} and take full advantage of:

- Convenient online submission

- Thorough peer review

- No space constraints or color figure charges

- Immediate publication on acceptance

- Inclusion in PubMed, CAS, Scopus and Google Scholar

- Research which is freely available for redistribution

Submit your manuscript at www.biomedcentral.com/submit
Ciomed Central 